\title{
Le Brésil, un pays qui s'affirme à l'extérieur parce qu'il est solide à l'intérieur
}

\section{Bernard Bret}

\section{(2) OpenEdition}

Journals

Édition électronique

URL : http://journals.openedition.org/echogeo/13850

DOI : $10.4000 /$ echogeo. 13850

ISSN : 1963-1197

Éditeur

Pôle de recherche pour l'organisation et la diffusion de l'information géographique (CNRS UMR 8586)

\section{Référence électronique}

Bernard Bret, « Le Brésil, un pays qui s'affirme à l'extérieur parce qu'il est solide à l'intérieur », EchoGéo [En ligne], Sur le Vif, mis en ligne le 12 juin 2014, consulté le 30 avril 2019. URL : http:// journals.openedition.org/echogeo/13850; DOI : 10.4000/echogeo.13850

Ce document a été généré automatiquement le 30 avril 2019.

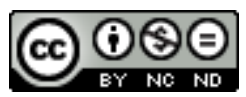

EchoGéo est mis à disposition selon les termes de la licence Creative Commons Attribution - Pas d'Utilisation Commerciale - Pas de Modification 4.0 International 


\title{
Le Brésil, un pays qui s'affirme à l'extérieur parce qu'il est solide à l'intérieur
}

\author{
Bernard Bret
}

1 Au moment où le Brésil s'apprête à recevoir en 2014 la Coupe du monde de football disons plutôt futebol pour nous mettre dans l'ambiance - puis en 2016 les Jeux olympiques, il peut être utile d'interroger ce pays sur les ambitions qu'il nourrit et sur sa capacité à tenir le rôle de premier plan auquel il aspire dans les affaires du monde. Les rencontres sportives qui vont s'y tenir le mettront sous les projecteurs de l'actualité : ce peut être l'occasion de prendre du recul, de remettre ce pays dans une perspective plus large pour mieux le comprendre, pour le voir dans un temps plus long et dans un contexte géographique examiné à plusieurs échelles.

2 Il convient en effet de voir ce pays d'un œil neuf. Depuis longtemps, le Brésil n'est plus seulement la terre des plantations de café ou de canne à sucre, même si ces productions et beaucoup d'autres font de lui un géant dans le commerce international des produits agricoles. C'est un pays industrialisé, mais le regard porté sur son industrie doit lui-même être attentif aux transformations du secteur et à la place que s'y sont faite les branches de haute technologie. Pays des vastes espaces quasi vides, certes, mais dont beaucoup sont desservis par des routes et déjà mis en valeur, et pays très majoritairement urbain. Pays finalement qui, avec Brasilia, donne à voir sa capacité à occuper son territoire et à en exploiter les richesses. Serait-ce tomber dans un cliché inutile que d'y souligner les contrastes entre l'opulence et la pauvreté, entre les quartiers chics et les favelas, entre les grands domaines agro-industriels et les paysans sans terre ? Ces contrastes existent. Ils donnent à voir les problèmes et les ambiguïtés de la croissance économique. Mais, si beaucoup reste à faire, il est indéniable que la course à une croissance accélérée sans considération des conséquences sociales et des effets environnementaux n'est plus à l'ordre du jour. Le pays a tourné le dos à ce qui, par abus de langage, avait été appelé le miracle brésilien à l'époque de la dictature militaire (1964 -1985). Le jeu normal des mouvements sociaux et leur expression dans la sphère politique font que le partage des 
richesses alimente aujourd'hui le débat public. Le pays a changé. Il poursuit son changement, et cela ne peut se faire sans convulsion, sans heurt ni conflit, même si la paix civile est garantie par le fonctionnement démocratique de l'État.

3 Le Brésil est-il devenu une grande puissance ? Est-il le pays dominant en Amérique latine ? Est-il solide ou fragile à l'intérieur de ses frontières?

\section{Une affirmation sur le plan international}

\section{À l'échelle mondiale...}

Depuis maintenant plus de trente ans que l'on parle des pays émergents, le Brésil est considéré comme l'un des principaux et figure dans l'acronyme BRIC à côté de la Russie, de l'Inde et de la Chine, souvent complété d'un S pour Afrique du Sud (BRICS), et même parfois d'un M pour Mexique (BRICSAM). La discussion pourrait être longue, et sans doute assez vaine, sur la pertinence de ce regroupement (la Russie est-elle vraiment un pays " émergent » alors qu'elle se reconstruit à partir des décombres de ce qui fut tout-demême la deuxième puissance mondiale ?) et sur les pays à y englober car des économies de gabarit plus modeste sont elles aussi en progrès rapide. Qu'il suffise ici de retenir que le Brésil connaît la croissance soutenue d'une économie déjà diversifiée, et que son PIB (2 252 milliards de dollars en 2012) le situe au 6 ou $7^{\mathrm{e}}$ rang dans le monde : peut-être le qualificatif d'émergé lui conviendrait-il mieux que celui d'émergent !

5 Le Brésil est aujourd'hui un acteur qui compte dans les affaires internationales et qui, comme l'Inde, revendique une place de membre permanent au Conseil de sécurité de l'ONU pour voir reconnu son poids démographique, économique et culturel. Il est vrai que l'ONU, mise en place au sortir de la Seconde Guerre mondiale, ne reflète plus les rapports de forces actuels et que maintenir exclus de son instance principale d'immenses aires culturelles et des pays devenus très importants dans les équilibres mondiaux n'est guère justifié. Pour crédibiliser cette candidature, le Brésil a accepté des responsabilités dans les opérations de l'organisation. Après avoir participé à des interventions en Angola et au Timor Oriental, il a pris en 2004 le commandement de la Mission des Nations unies de stabilisation en Haïti (MINUSTAH), puis, en 2011, il a été mis à la tête des opérations maritimes de la Force intérimaire des Nations unies au Liban (FINUL). Sa diplomatie est très active en Afrique, dont le président Luiz Inacio Lula da Silva a visité pas moins de 23 États, avec une attention particulière portée aux pays lusophones. Le continent africain reçoit une bonne part des aides qu'il accorde aux pays en développement, et cette coopération Sud-Sud, à elle seule, dit bien que le Brésil bouscule les schémas traditionnels des relations Nord-Sud. Consolidant cette présence diplomatique, les intérêts économiques du Brésil sont représentés dans le monde entier à travers ses firmes multinationales. Là encore, on est habitué à parler des firmes étrangères opérant au Brésil. Or, il faut aussi s'habituer aux firmes brésiliennes opérant hors du Brésil. La plus connue est Vale, dans l'extraction et la métallurgie, née en 1997 de la privatisation de la CVRD (Companhia do Vale do Rio Doce) et qui emploie environ 150000 salariés dans plus de 30 pays. Il en est d'autres, telles Petrobras dans les hydrocarbures et, moins connue en Europe, Odebrecht dans le BTP. 


\section{... comme à l'échelle latino-américaine}

6 Être fort dans le monde suppose de l'être aussi dans son aire culturelle. Aussi, en Amérique latine, et bien qu'il se distingue de ses voisins hispanophones, le Brésil a pu et a su se poser en concurrent des États-Unis dans la tendance à l'intégration des économies américaines. L'idée que le Nouveau Monde constitue une entité qui doit s'organiser est ancienne. Mais, dès 1823 et la Déclaration Monroe, cette organisation a toujours été asymétrique avec, d'un côté, une puissance dominante, et de l'autre des pays dominés. Très vite, et face aux droits que Washington s'octroyait dans son arrière-cour, la formule de "l'Amérique aux Américains» a été dénoncée par les Latino-Américains comme signifiant «l'Amérique du Sud aux Américains du Nord». Si tous les pays du continent ont acté leur solidarité de défense en 1947 dans le Traité interaméricain d'assistance réciproque - TIAR - et si en 1948 a été créée l'Organisation des États américains - OEA - il n'empêche qu'une concurrence s'est engagée entre deux Amériques, l'une anglo-saxonne menée par la puissance mondiale que sont les États-Unis, l'autre latino-américaine menée par l'autre géant qu'est le Brésil.

7 C'est dans les années 1960 que les premières intégrations ont vu le jour en Amérique latine, fragiles et regroupant des partenaires modestes en Amérique centrale, dans les Caraïbes et dans les pays andins. Une étape plus importante a été franchie en 1991 quand le Brésil, par le Traité d'Asuncion signé avec l'Argentine, le Paraguay et l'Uruguay, a créé le Mercosul - ou Mercosur en espagnol - Marché commun du Sud, d'abord zone de libreéchange, puis union douanière après l'adoption d'un tarif extérieur commun en 1994 (Protocole d'Ouro Preto). Avant d'examiner plus avant les résultats, au demeurant inégaux, de cette association, il faut remettre sa naissance dans son contexte géopolitique pour mieux comprendre les ambitions brésiliennes. En 1990, soit un an avant le Traité d'Asuncion, le président Bush avait lancé une « Initiative pour les Amériques » et dit son souhait de voir se mettre en place une intégration à l'échelle continentale à concrétiser dès 2005 dans une Zone de libre-échange des Amériques - ZLEA - encore appelée Association de libre commerce des Amériques - ALCA. Un vaste marché étendu de l'Alaska à la Terre de Feu ne pouvait manquer d'inquiéter les pays latino-américains, car le projet risquait fort de renforcer la suprématie des États-Unis. Le Mercosul constituait donc une résistance au projet de Washington.

8 En s'affichant au Sud comme un pôle de rééquilibrage face aux prétentions des États-Unis, le Brésil prenait alors une direction contraire à celle dans laquelle s'engageait le Mexique. En 1992, ce pays signait en effet avec les États-Unis et le Canada (lesquels étaient déjà liés par un accord datant de 1989) un accord créant l'Accord de libre-échange nordaméricain. Avec l'ALENA (en anglais : NAFTA), il tournait le dos à son aire culturelle, mais peut-être sa proximité avec les États-Unis (« Pauvre Mexique, si loin de Dieu et si près des États-Unis », disait déjà Porfirio Diaz il y a plus d'un siècle) lui interdisait-elle d'autres choix. Toujours est-il que devenait évidente la concurrence de deux puissances pourtant amies, l'une grande puissance et l'autre puissance émergente : d'un côté, les États-Unis qui ne faisaient pas mystère de voir dans l'ALENA l'amorce de l'ALCA, de l'autre le Brésil qui entendait faire du Mercosul une alternative crédible à ce projet.

9 Le Mercosul a connu des hauts et des bas. Les échanges commerciaux entre les partenaires se sont d'abord accrus, signe d'une attirance communautaire freinée ensuite par la crise argentine des années 2000. Le Mercosul a enregistré en tous les cas assez de 
succès pour attirer. L'association de plusieurs pays a montré son attractivité. Le Venezuela en est membre depuis 2012 et la Bolivie attend à cette date la ratification de sa demande d'adhésion. Pour les raisons dites plus bas, il n'est pas sûr que l'inclusion de ces deux pays change sensiblement la donne géopolitique latino-américaine. Il est plus important ici de s'interroger sur la place du Brésil dans le Mercosul et pourquoi c'est un motif d'inquiétude pour ses voisins. Fait essentiel, le Mercosul est une intégration asymétrique. La comparaison avec l'Alena ne peut manquer de venir à l'esprit, quand bien même les écarts de développement entre les États-Unis et le Mexique sont beaucoup plus forts que ceux existant entre le Brésil et ses partenaires. Il demeure que, en termes de PIB (l'indicateur a tous les défauts du monde, mais il n'est pas dépourvu de signification), le Brésil, c'est 5 fois l'Argentine, 46 fois l'Uruguay ... et 90 fois le Paraguay ! Par ailleurs, les intérêts brésiliens sont beaucoup plus présents chez ses voisins que l'inverse. Mais, finalement, cette asymétrie n'est pas nécessairement contradictoire avec la réussite du Mercosul car elle dit une certaine complémentarité des pays membres, comme si le Brésil, ou plus exactement sa région Sudeste et surtout Sao Paulo, était le « Nord » économique du Mercosul, lequel répliquerait à son échelle les relations Nord-Sud de la planète comme le fait l'Alena. Cela signifie aussi, autre asymétrie, que le Brésil a moins besoin du Mercosul que ses partenaires : effet de taille comme de niveau de développement, un pays comme le Paraguay dépend du Mercosul pour les deux tiers de ses exportations, quand le Brésil n'en dépend que pour le dixième. Il n'est pas confortable de dépendre fortement d'une puissance qui, elle, peut se passer de vous sans dommage ! Or, c'est un peu ce qui se passe quand le Brésil, faute qu'aboutissent les conversations commerciales commencées dès 1995 entre le Mercosul et l'Union européenne, engage des discussions directement d'État à État et signe, par exemple, un partenariat stratégique avec la France. Le Mercosul est finalement trop petit pour les ambitions du Brésil (les échanges à l'intérieur du Mercosul ne font que $0,3 \% \mathrm{du}$ commerce mondial!). Toutefois, les enjeux politiques interfèrent avec les questions économiques, car, si le Brésil peut se passer de ses partenaires pour ses échanges, il a besoin d'eux pour soutenir ses revendications à l'ONU. Par ailleurs, il ne faut pas oublier que, créé par des pays qui avaient depuis peu chassé les militaires du pouvoir, le Mercosul a une dimension politique contenue dans une clause démocratique précieuse entre toutes: celle d'être une assurance mutuelle contre le retour des dictatures ${ }^{2}$.

Dès le début de la décennie suivante, en fait, ont été portés en Amérique latine deux projets concurrents, l'un piloté par le Brésil et l'autre par le Venezuela. Recevant les présidents sud-américains pour un sommet en 2000, Brasilia a lancé l'Initiative d'intégration de l'infrastructure régionale d'Amérique du Sud - IIRSA. Ce vaste programme touchant les transports, l'énergie et les télécommunications concrétise d'une façon pragmatique l'idée d'intégration en dessinant un aménagement pensé à l'échelle de tout le sous-continent... mais avantageux surtout pour le Brésil. Avec les liaisons vers la Bolivie et la côte du Chili, ainsi que les voies fluviales des rios Parana, Paraguay et Uruguay, c'est la région vitale du Sudeste qui regarde vers l'Ouest, s'ouvre une fenêtre sur le Pacifique et renforce par ailleurs son influence dans le bassin de la Plata. D'autres travaux, il est vrai, facilitent les liaisons de l'Amazonie brésilienne avec le Pérou, l'Équateur et le Venezuela, mais l'essentiel est bien ce que l'IIRSA désigne sous le nom "d'Axe inter-océanique central », qui offre à Sao Paulo un outil pour consolider son emprise et l'étendre au-delà des frontières nationales. 
11 Peu après, en 2004, le Mercosul se rapprochait de la Communauté andine des nations pour fonder la Communauté sud-américaine des nations, devenue en 2008 l'Union des nations sud-américaines - Unasur. Ce nouvel organisme se superpose aux précédents sans les supprimer, ce qui n'est pas fait pour simplifier un paysage institutionnel passablement complexe. Il vise à faire coopérer les États membres dans les domaines de l'économie (l'objectif demeure toujours de libérer davantage les échanges commerciaux, raison pour laquelle l'Unasur a pris sous sa responsabilité le programme IIRSA en 2011), des questions sociales, de la culture. Il veut être aussi un espace de dialogue politique. C'est surtout sur ce terrain politique que la divergence est perceptible entre le Brésil et le Venezuela, initiateur de l'Alliance bolivarienne pour les Amériques - ALBA. L'acronyme affiche à lui seul l'objectif de Caracas : proposer aux pays latino-américains une alternative au projet de l'ALCA. Ce dernier peut, c'est vrai, être déjà considéré comme abandonné quand, en 2005, les présidents Hugo Chavez pour le Venezuela, Fidel Castro pour Cuba, Daniel Ortega pour le Nicaragua et Rafaël Correa pour l'Équateur signent à La Havane le traité donnant naissance à l'ALBA. Mais la lutte contre ce que représentait le projet de l'ALCA reste à l'ordre du jour car les États-Unis, contournant l'échec de leur projet initial, signaient alors des accords bilatéraux avec les pays latino-américains pour arriver au libre-échange qu'ils n'avaient pas pu imposer par un traité d'ensemble. Dans l'ALBA, la référence à Bolivar dit bien la volonté d'intégration, mais contre le modèle néo-libéral et dans un souci de développement endogène attentif à la pauvreté et à l'exclusion sociale. Toutefois, cette référence ne veut pas dire que l'organisation ait vraiment bousculé l'ordre international établi et ait dépassé une fonction tribunicienne. Malgré ces divergences et le fait que le Mexique ait lié son sort à celui de son puissant voisin, une structure est née en 2011, la Communauté d'États latino-américains et Caraïbes (sorte d'OEA - $2+1$, c'est-à-dire - États-Unis et Canada et $+\mathrm{Cuba})$, qui veut consolider le rapprochement d'États se reconnaissant une proximité culturelle et une convergence d'intérêts ${ }^{3}$.

12 La rapide succession des accords entre les États et l'avalanche de sigles qui en résulte ne doivent pas masquer la tendance lourde en ce qui concerne notre sujet: sans se placer dans une confrontation hostile aux États-Unis, le Brésil entend constituer le pôle d'un nouvel équilibre du continent, et la suprématie qu'il exerce sur ses partenaires latinoaméricains lui permet de se positionner efficacement dans l'économie mondialisée.

\section{Les moyens de l'ambition}

13 Une interrogation découle de ce qui précède : le Brésil a-t-il les moyens de ses ambitions ?

\section{Des atouts économiques...}

14 L'économie donne certes quelque inquiétude avec un taux de croissance ralenti ces dernières années (2,7 \% en 2011 et $0,9 \%$ en 2012), loin derrière les performances de l'Inde et de la Chine. Cependant, on voudrait ici mettre en évidence les tendances lourdes plutôt que les fluctuations conjoncturelles. Apparaissent alors de solides atouts. C'est d'abord la relative solidité de sa monnaie. Bien sûr, le real n'échappe pas plus que d'autres devises aux variations de cours. Il traverse d'ailleurs une passe difficile en 2013 et 2014 comme d'autres monnaies de pays émergents (l'Afrique du Sud, l'Inde, la Turquie). Mais cela fait 
maintenant plus de 20 ans que le real est une monnaie crédible. C'est Fernando Henrique Cardoso, alors ministre des finances, qui avec son plan real de 1993 (contrôle de la masse monétaire, réduction des dépenses publiques et désindexation des prix et des salaires) a mis un terme à l'inflation galopante. Le succès de cette très difficile opération a valu à son promoteur d'être ensuite élu à la Présidence de la République.

Deuxième atout, bien connu des géographes, les ressources naturelles. Le dire ne consiste pas à tomber dans un raisonnement déterministe, mais à constater l'évidence. Sur son immense territoire ( 15 fois la France), le Brésil dispose d'abord de réserves foncières considérables, à l'Ouest bien sûr, mais pas seulement, avec en plus, une forte marge d'intensification dans les régions mises en valeur depuis longtemps. Les travaux d'Hervé Théry sur la frontière agricole et la progression du soja sont assez convaincants pour qu'il soit inutile d'y revenir ici, sinon pour rappeler simplement que le Brésil a les moyens de devenir la ferme du monde. Les ressources minérales sont elles aussi énormes, notamment en minerai de fer et minerais non ferreux, telle la bauxite. Sur le plan énergétique, le potentiel hydro-électrique connu de longue date est de mieux en mieux exploité. Mais le fait marquant de ces dernières années concerne les hydrocarbures. Devenu autosuffisant en pétrole depuis 2006 et produisant en 2014 plus de 100 millions de tonnes, le Brésil a découvert l'année suivante un énorme gisement sous-marin, dit «présal », sous $2000 \mathrm{~m}$ d'eau et $5000 \mathrm{~m}$ de sédiments, au large des côtes de Santa Catarina à l'Espirito Santo. Des entreprises privées, notamment la Shell et Total, et aussi des entreprises chinoises se sont associées à l'entreprise publique Petrobras pour l'exploration et l'exploitation de cette manne. Déjà treizième producteur mondial (avant des pays dont le nom seul évoque l'or noir, tels que la Norvège, l'Algérie, la Libye et le Qatar !), le Brésil peut devenir dans les prochaines années une des premières puissances pétrolières.

16 Comme dans d'autres pays émergents et grâce aux cours élevés des matières premières, on assiste donc au Brésil à une reprimarisation de l'économie et, en particulier, du commerce extérieur. Mais le terme de «reprimarisation» ne doit pas être interprété négativement. Il est vrai que les échanges de produits manufacturés sont aujourd'hui déficitaires, ce qui n'est pas nécessairement inquiétant car, dans ce poste d'échange, il faut distinguer les biens d'équipement et les biens de consommation : acheter des biens d'équipement pour accroître et moderniser son parc industriel et devenir plus fort dans la fourniture de biens de consommation n'est pas un mauvais calcul. Surtout, les exportations de produits manufacturés ont augmenté en valeur absolue, passant de 16 milliards de dollars en 1990 à 82 milliards en 2012. En revanche, leur part dans les ventes à l'étranger a baissé, passant de $52 \%$ en 2005 à $34 \%$ en 2012, et cela à cause d'une croissance plus forte des produits minéraux (18\% des exportations en 2005 et $30 \%$ en 2012 ) et des produits agricoles ( $30 \%$ des exportations en 2005 et $36 \%$ en 2012). Ces produits minéraux et agricoles assurent des rentrées de devises et permettent au Brésil d'avoir de grosses réserves de change. Si tel n'était pas le cas, on ne parlerait pas de « reprimarisation ». Utiliser ce mot n'est donc pas suggérer que le Brésil reculerait sur le plan industriel, mais dire qu'en plus de son industrie, il a une puissante activité extractive et une puissante agriculture. Ce n'est pas une faiblesse, mais une force.

17 Un autre atout se trouve dans la maitrise de technologies avancées. On sait que la firme Embraer (Empresa Brasileira de Aeronautica) s'est imposée dans le secteur aéronautique, non pas en prétendant concurrencer Boeing ou Airbus, mais en choisissant dans un premier temps le créneau plus modeste et plus réaliste des petits appareils à hélices, 
avant de monter en gamme et de produire des jets de taille moyenne vendus aujourd'hui aux plus grandes compagnies de transport aérien. Sao José dos Campos, où elle est installée avec d'autres entreprises de haute technologie, constitue la technopole la plus importante du pays. Mais il est d'autres savoir-faire où le Brésil tient les premières places. On en mentionnera deux, liées l'une et l'autre au problème énergétique. Ce sont d'abord les biocarburants. En réponse aux deux chocs pétroliers, le Brésil a fait le pari de l'alcoolcarburant comme produit de substitution à une énergie fossile qui, à l'époque, faisait défaut sur le sol national. Il a acquis en la matière une expérience qui n'a pas d'égale dans le monde. Certes, l'extension des superficies en canne à sucre et la consolidation du modèle agro-industriel qui est allée de pair posent des problèmes sociaux et environnementaux qui ne doivent pas être ignorés. Néanmoins, sur le plan de la maitrise technologique, le Brésil a pris une avance considérable: usines de traitement capables d'ajuster leur production en fonction du marché et de fournir soit du sucre, soit de l'alcool-carburant, moteur flex-fuel fonctionnant indifféremment à l'essence ou à l'alcoolcarburant, recherches en cours sur le biodiesel à partir du soja et du ricin. C'est ensuite la recherche d'hydrocarbures en off-shore sous grande profondeur. Il a été dit la découverte du champ pétrolier nommé pré-sal. Dans le même temps qu'il mettait au point des énergies de substitution, le Brésil s'engageait en effet dans l'exploration du pétrole sousmarin. Non seulement cette recherche a porté ses fruits, mais encore elle a été l'occasion de grands progrès technologiques. Petrobras a acquis en cette matière une expertise reconnue mondialement.

\section{... et des atouts sociaux}

La puissance d'un pays ne repose pas seulement sur son économie. Compte aussi sa cohésion sociale, laquelle tient à un consensus sur la dynamique sociale à l'œuvre. Il ne s'agit pas d'un consensus sur la structure sociale existante, au demeurant impossible et non souhaitable car ce serait accepter des injustices criantes, mais sur la façon dont cette société évolue. Pour dire les choses d'une façon sans doute un peu simplificatrice mais qui va à l'essentiel, le Brésil est passé de la croissance au développement.

Deux faits majeurs caractérisent l'évolution de la société brésilienne depuis une quinzaine d'années : le recul de la pauvreté et l'essor des classes moyennes.

Le président Luiz Inacio Lula da Silva au pouvoir de 2002 à 2010 est issu du monde syndical et du Parti des travailleurs. Il a su répondre aux attentes populaires tout en gardant ou en gagnant la confiance des classes moyennes, fût-ce au prix de compromis politiques parfois douteux ${ }^{4}$. Les faits sont là : les Brésiliens les plus défavorisés ont bénéficié de prestations sociales substantielles. Il est juste de rappeler que ces prestations ne datent pas toutes de l'arrivée au pouvoir du Parti des travailleurs. Fernando Henrique Cardoso, déjà mentionné pour sa victoire sur l'inflation et qui a exercé deux mandats présidentiels, en avait créé certaines. Mais, dès son discours d'investiture, le président Lula donnait un coup d'accélérateur à cette politique sociale en lançant le plan Faim zéro. Aujourd'hui, les aides diverses (bourse scolaire, aide à l'achat des bouteilles de gaz, bourse d'alimentation) sont regroupées dans une Bolsa Familia, une Bourse famille versée à plus de 12 millions de familles, pour un montant moyen de 150 reais, soit environ 50 euros par mois. Somme qui pourrait apparaître dérisoire vue d'Europe, mais qui est vitale pour les plus pauvres au Brésil. Cette aide est modulée selon le nombre d'enfants à charge, sous condition que les enfants aillent à l'école et suivent les programmes de 
vaccination. C'est faire d'une aide matérielle une incitation forte à la scolarisation et au suivi médical. Si l'on ajoute que la somme est versée directement aux mères de famille, c'est aussi un facteur important pour la promotion des femmes. Cette allocation a fait sortir de la misère des millions de Brésiliens.

Or, dans le même temps, la classe moyenne s'est considérablement étoffée. Les statistiques brésiliennes distinguent 5 classes de revenus de $\mathrm{A}$ à $\mathrm{E}$. Entre d'une part les riches (classe A) et les gens aisés (classe B) et, d'autre part, les petites revenus (classe D) et les pauvres (classe E), la classe dite $C$ désigne la classe moyenne. Ce terme mérite explicitation. Cette classe moyenne n'a pas, en effet, les revenus de la catégorie sociale ainsi nommée en Europe. Elle est également beaucoup plus modeste de ce que, naguère, on appelait classe moyenne au Brésil et qui, très au-dessus de la moyenne d'alors, correspondait davantage à ce qui constitue aujourd'hui la classe B. Cette classe moyenne entendue comme la classe $C$ se définit par des seuils de revenus périodiquement mis à jour en fonction de l'évolution du coût de la vie. En février 2014, elle correspond à un revenu mensuel par tête situé entre un minimum de 320 reais et un maximum de 1120 reais. Elle est donc fortement hétérogène, mais le trait commun de ses membres est qu'ils ont un pouvoir d'achat supérieur à la couverture des besoins de première nécessité, il est vrai à des degrés inégaux. Or cette catégorie de population représente aujourd'hui plus de la moitié de la population totale: le Brésil est devenu un pays de classe moyenne majoritaire! Cette proportion augmente chaque année. En 1992, elle était de $35 \%$. En 2009, la barre des $50 \%$ était atteinte. Puis, ce fut $52 \%$ en 2010 et $54 \%$ en 2014 . D'une société en pyramide, avec une base très large de pauvres et où les effectifs se réduisaient très vite vers le haut au fur et à mesure qu'augmentaient les revenus, on passe à une société dont la silhouette change sensiblement: une minorité de pauvres à la base, une majorité de classes moyennes au milieu, une minorité de riches au sommet. On pourrait évidemment discuter la limite supérieure de cette classe de revenus, et plus encore le seuil qui la borne vers le bas. Ce serait s'interroger du coup sur la pertinence du terme « classe moyenne » appliquée à ses catégories les plus modestes. Les spécialistes brésiliens en sont conscients et parlent de "nouvelle classe moyenne" pour désigner ces populations dont les conditions de vie ne sont pas encore satisfaisantes, mais qui sont sorties de la précarité et qui accèdent à de nouveaux modes de consommation.

Plusieurs explications rendent compte de ce gonflement des classes moyennes. C'est la croissance économique, cause autant que conséquence, qui a permis de créer pas moins de 12 millions d'emplois entre 2003 et 2010. C'est aussi la fin de l'inflation galopante qui, avant le plan real, avait des effets sociaux dévastateurs : l'amélioration des conditions de vie des Brésiliens qui a été très sensible au cours des deux mandats du président Lula doit beaucoup aussi à l'assainissement monétaire réussi avant lui. Mais le moteur principal est surtout la hausse des salaires, témoin elle-même d'un nouveau rapport de forces entre les partenaires sociaux. En 2002, le salaire minimum se montait à 200 reais, soit environ 87 euros. Il est passé à 510 reais en 2010, soit 219 euros, et a continué ensuite sur la même tendance, atteignant 724 reais en janvier 2014, soit 240 euros. Si ce niveau est encore médiocre, il faut tenir compte que la conversion en euros donne une image minorée du pouvoir d'achat. Il faut surtout retenir que la tendance va dans le bon sens pour corriger des injustices inscrites dans le temps long et permettre un rattrapage salarial après la contention des basses rémunérations qui avait eu lieu durant la dictature militaire (1964 1985). Il faut enfin noter que la hausse du salaire minimum tire vers le haut les autres 
salaires et a donc un impact indirect sur beaucoup plus de travailleurs que ceux concernés directement.

Le gonflement des effectifs de la classe moyenne a de gros effets sur l'économie. L'augmentation d'une demande solvable, cela veut dire plus de consommation de biens de longue durée, de biens culturels, d'éducation, de soins de santé, et accès plus facile au crédit bancaire. Sur le plan politique, ce n'est pas sans conséquence. Cela dit aussi sans doute plus d'adhésion à l'orientation social-démocrate de la politique suivie, en dépit de l'étiquette du parti au pouvoir ${ }^{5}$.

Dans ces conditions, on comprend que Dilma Rousseff ${ }^{6}$ ait succédé à Lula. Son arrivée au Palais du Planalto symbolise les progrès de la démocratie. Son action la concrétise : lutte contre la corruption, ce mal endémique dont le Parti des travailleurs lui-même n'a pas été exempt, et poursuite de la politique sociale (l'amplification du programme Minha Vida, Minha Casa ${ }^{7}$ en faveur du logement populaire en est un exemple). Déjà, l'élection de Lula avait été plus que la victoire de la Gauche. C'était l'arrivée à la Présidence d'un homme du peuple, dans une fonction jusqu'alors toujours exercée par les élites de la fortune ou du savoir, quand elle n'était pas confisquée par des militaires factieux. En voyant l'un des leurs au sommet de l'État, les masses populaires ressentent légitimement que l'État, c'est aussi leur affaire, l'affaire des "petits » et pas seulement des " gros », et du coup chacun prenait mieux conscience de sa qualité de citoyen et des droits afférents. Ensuite, avec l'élection d'une femme en la personne de Dilma Rousseff, le Brésil montrait la maturité de sa démocratie et donnait à voir un nouveau symbole des progrès que réalise sa société.

\section{Pour conclure}

Est-ce à dire que tout va bien? Ce serait sottise de le prétendre, et une analyse plus poussée que ce rapide survol montrerait les graves défis à résoudre: une croissance urbaine souvent anarchique, des favelas qui, malgré d'incontestables améliorations, signalent une crise aiguë du logement et se trouvent pour certaines sous le contrôle des narco-trafiquants, une violence qui pousse à une surenchère sécuritaire, avec notamment les résidences fermées qui fragmentent l'espace urbain et menacent la ville en tant que cité, des paysans sans terre à la campagne qui dénoncent la concentration opérée par les grandes entreprises agro-industrielles, des atteintes à l'environnement aussi bien dans les régions industrialisées que dans les régions de l'Ouest en cours de mise en valeur, le sort inquiétant des Indiens malgré les protections juridiques dont ils sont l'objet et les territoires qui leur sont réservés. L'opinion pointe en particulier les faiblesses du système d'enseignement et des services de santé. Elle s'indigne que la corruption gangrène l'action des pouvoirs publics.

On pourrait donc décrire une situation explosive. Cette description serait aussi fausse que celle qui gommerait les difficultés. Ce qui est vrai, c'est que le Brésil a les moyens économiques et politiques d'affronter ses difficultés. C'est dans cette perspective que doivent être lues les manifestations de rue survenues en juin 2013 dans un grand nombre de villes. D'abord lancées contre les augmentations des tarifs des transports urbains, ces protestations ont visé aussi l'insuffisance de nombreux services publics et ont dénoncé les dépenses jugées excessives engagées pour la Coupe du monde et les Jeux olympiques. Le pouvoir a dû écouter ce mécontentement et se trouve dans l'obligation d'y répondre. Tout cela est-il un mauvais ou un bon signe? Le fait que la classe moyenne manifeste, car c'était plutôt la petite classe moyenne que les classes populaires, dit une montée de sa 
conscience politique autant que son goût pour une consommation qui est pour elle chose nouvelle. Qu'elle conteste l'emploi de l'argent public dans des dépenses de prestige plutôt que pour des usages sociaux, cela mérite considération, surtout dans un pays où, croyaiton, le ballon rond devait être mis a priori au-dessus de toute critique. Qu'elle puisse le faire dans la rue, cela signifie aussi qu'est effectif le droit de manifestation. Que le pouvoir ne voie pas dans la répression la seule réponse à apporter, cela montre que la démocratie est validée comme le système légitime du fonctionnement social. Là se trouve sans doute la principale force du Brésil d'aujourd'hui.

\section{BIBLIOGRAPHIE}

L'autre continent du football, Cahiers des Amériques latines, $\mathrm{n}^{\circ}$ 74, avril 2014.

Dabène O. (dir), 2006. Atlas de l'Amérique latine. Paris, Autrement, 80 p.

Dabène O., Louault F., 2013. Atlas du Brésil. Paris, Autrement, 93 p.

Droulers M., Broggio C., 2013. Le Brésil. Paris, PUF, coll. Que sais-je ? $3^{\circ}$ éd., 128 p.

Rouquié A., 2006. Le Brésil au XXI siècle, Naissance d'un nouveau grand. Paris, Fayard, 409 p.

Salama P., 2012. Les économies émergentes latino-américaines entre cigales et fourmis. Paris, A. Colin, coll. U, 225 p.

Théry H., 2012. Le Brésil. Paris, A. Colin, $6^{\circ}$ éd., 296 p.

Théry H., 2014. Le Brésil, pays émergé. Paris, A. Colin coll. Perspectives géopolitiques, 296 p.

Théry H., Aparecida De Mello N., 2003. Atlas du Brésil. Paris, CNRS-Libergéo-La Documentation française, coll. Dynamiques du territoire, $452 \mathrm{p}$.

\section{NOTES}

1. C'est ce qu'affiche le livre d'Hervé Théry Le Brésil, pays émergé paru quelques semaines après la rédaction de cet article.

2. Cette clause a permis de faire échec à une tentative de coup d'État au Paraguay en 1999 et de suspendre ce même État en 2012, à la suite de la destitution illégale du président Fernando Lugo.

3. Voir Olivier Dabène : La quatrième vague de régionalisme. In $\mathrm{C}$. Quenan et $\mathrm{S}$. Velut, Les enjeux du développement en Amérique latine, AFD et IDA, janvier 2014.

4. La dispersion des partis à la Chambre des députés a conduit à des compromis avec d'autres forces politiques que le PT, et, plus grave, à la corruption de certains élus. En 2005, le scandale dit du mensalao, c'est-à-dire de la mensualité, a mis au jour un système qui achetait les votes de certains députés de l'opposition. De nombreux hommes du PT ont été compromis dans l'affaire, dont Jorge Dirceu, à l'époque des faits ministre de la Maison civile (c'est-à-dire non pas le Premier ministre, mais le premier des ministres, associé directement à l'action du Président) poussé à la démission et plus tard condamné au pénal. 
5. Le Parti des travailleurs a conservé son nom, mais a élargi sa base électorale. Il conduit aujourd'hui une politique que l'on peut qualifier de social-démocrate : forte implication de l'État et politique sociale dans une économie de marché. Il faut signaler que le PSDB, Parti de la socialdémocratie brésilienne, est celui du prédécesseur de Lula, Fernando Henrique Cardoso, aujourd'hui dans l'opposition et situé au centre, sinon au centre-droit de l'échiquier politique.

6. Economiste qui a connu la prison et la torture sous le régime militaire, cette femme d'appareil a longtemps travaillé dans l'ombre de son prédécesseur.

7. Ma vie, ma maison.

\section{RÉSUMÉS}

En accueillant des manifestations sportives de prestige, le Brésil affiche sa volonté de s'affirmer sur la scène internationale. Il le fait à l'échelle mondiale comme à l'échelle latino-américaine et exerce une suprématie sur les pays voisins avec lesquels il conduit une politique d'intégration. Ses ambitions sont rendues crédibles par ses performances économiques et plus encore par le renforcement de sa cohésion sociale, rendu possible par le recul de la pauvreté et l'essor des classes moyennes.

Welcoming some important sporting events, Brazil wishes to get a more important international role all over the world as well as in South America, and acts as a leader on the neighbouring countries with which it has a intergration policy. Its ambitions become real with its economic performance, and with the reinforcement of its social cohesion, made possible by less poverty and the growness of mid-classes.

\section{INDEX}

Mots-clés : Brésil, classes moyennes, intégration, Mercosul, pauvreté, pays émergent, ressources Keywords : Brazil, mid-classes, integration, Mercosul, poverty, emerging country, ressources

\section{AUTEUR}

\section{BERNARD BRET}

Bernard Bret, bernard.bret12@wanadoo.fr, est Professeur émérite à l'Université Jean Moulin Lyon 3, membre de l'UMR 5600 EVS. 